\title{
T cell-activation in neuromyelitis optica lesions plays a role in their formation
}

\author{
Maria Pohl', Naoto Kawakami ${ }^{2,3}$, Maja Kitic ${ }^{1}$, Jan Bauer ${ }^{1}$, Rui Martins ${ }^{1}$, Marie-Therese Fischer ${ }^{1}$, Joana Machado-Santos ${ }^{1}$, \\ Simone Mader ${ }^{4}$, Joachim W Ellwart ${ }^{5}$, Tatsuro Misu ${ }^{6}$, Kazuo Fujihara ${ }^{6}$, Hartmut Wekerle², Markus Reindl ${ }^{4}$, \\ Hans Lassmann ${ }^{1}$ and Monika Bradl ${ }^{1 *}$
}

\begin{abstract}
Background: Neuromyelitis optica (NMO) is an inflammatory demyelinating disease of the central nervous system (CNS), which is characterized by the presence of pathogenic serum autoantibodies against aquaporin 4 (AQP4) in the vast majority of patients. The contribution of T cells to the formation of astrocyte destructive lesions is currently unclear. However, active human NMO lesions contain CD4 ${ }^{+}$T-lymphocytes expressing the activation marker Ox40, and the expression is more profound compared to that seen in MS lesions of comparable activity. Therefore, we analyzed the role of T-cell activation within the CNS in the initiation of NMO lesions in an experimental model of co-transfer of different encephalitogenic T-cells and human AQP4 antibody containing NMO immunoglobulin $(\mathrm{NMO} \lg \mathrm{G})$. We further studied the expression of the T-cell activation marker Ox40 in NMO and multiple sclerosis lesions in different stages of activity.

Results: All encephalitogenic T-cell lines used in our experiments induced brain inflammation with a comparable extent of blood brain barrier damage, allowing human NMO lgG to penetrate into the brain and spinal cord tissue. However, astrocyte destructive NMO lesions were only seen with T-cells, which showed signs of activation in the lesions. T-cell activation was reflected by the expression of the activation marker Ox40 and pronounced production of $Y$-IFN, which was able to increase the production of complement proteins and of the Fc gamma III receptor (Fcgr3) and decreased production of complement inhibitory protein Factor $\mathrm{H}$ in microglia.
\end{abstract}

Conclusions: Our data indicate that local activation of T-cells provide an inflammatory environment in the CNS, which allows AQP4 auto-antibodies to induce astrocyte destructive NMO-like lesions.

Keywords: Neuromyelitis optica, T cell activation, Aquaporin 4, Lesion, IFN- $\gamma$

\section{Background}

Neuromyelitis optica (NMO) is an astrocytopathic disease of the central nervous system (CNS) characterized by optic neuritis, transverse myelitis, and - in the vast majority of patients - by the presence of specific autoantibodies, the so-called NMO-IgGs [1]. These antibodies are directed against aquaporin 4 (AQP4), a water channel particularly enriched on astrocytic processes at the glia limitans $[2,3]$, and they are pathogenic. They can bind to AQP4 on the surface of astrocytes, fix complement, and initiate complement-mediated destruction of these cells $[4,5]$. Based on earlier findings in NMO models it

\footnotetext{
* Correspondence: monika.bradl@meduniwien.ac.at

'Department of Neuroimmunology, Center for Brain Research, Medical

University Vienna, Spitalgasse 4, Vienna A-1090, Austria

Full list of author information is available at the end of the article
}

became clear that the presence of serum NMO-IgG is not sufficient to initiate astrocyte-destructive lesions [5], similar to the situation in NMO patients, who can be NMO-IgG seropositive for many years without showing clinical evidence of NMO [6]. Interestingly, even the presence of NMO-IgG and complement components in the CNS parenchyma is insufficient to promote the formation of large experimental NMO lesions, as seen when NMO-IgG and complement is injected directly into the striatum of mice with low numbers or abolished effector functions of circulating neutrophils [7], or when NMOIgG and complement gain access to the brain through a blood-brain barrier that is developmentally leaky $[5,8]$ or was rendered leaky due to the action of toxins [8] or to the intra-cerebral injection of cytokines [9]. All these 
observations clearly suggested that additional effector mechanisms are needed to initiate/promote astrocytedestructive lesions. Further studies revealed that these mechanisms are triggered by CNS inflammation. In NMO patients, active NMO lesions are characterized by complement deposition on and subsequent destruction of astrocytes, and have a pronounced inflammatory component as evidenced by the presence of $\mathrm{T}$ cells, many neutrophils, macrophages/activated microglia cells and some eosinophils $[10,11]$. In experimental NMO, lesions were induced by myelin basic protein (MBP)specific T cells in NMO-IgG seropositive Lewis rats, and faithfully reproduced essential features of active lesions in NMO patients $[4,5]$. Since the antigen recognition of $\mathrm{T}$ cells found in NMO lesions is still unknown, the findings in experimental NMO raise important questions: Are all CNS antigen-specific T cells similarly able to initiate astrocyte-destructive lesions in NMO-IgG seropositive hosts? And if not, what are the requirements for T cells to do so? These questions were answered in the current study.

\section{Methods \\ NMO IgG preparation}

The IgG preparation of the NMO patient J0 used has been extensively characterized before [5], and is termed NMO-IgG throughout this article. Its use was approved by the Ethics Committee of Tohoku University School of Medicine (No. 2007-327).

\section{Human tissue samples}

Autopsy CNS tissue of $6 \mathrm{NMO}$ and 10 MS patients (Table 1) from paraffin blocks and sections archived in the Center of Brain Research, Medical University Vienna, Austria or in Tohoku Medical University was used. The study was approved by the Ethical Committee of the Medical University of Vienna (EK. No. 535/2004 and 087/01/2012).

\section{Animals}

8 week-old Lewis rats from Charles River Wiga (Sulzfeld, Germany) were used. They were housed in the Decentral Facilities of the Institute for Biomedical Research (Medical University Vienna) under standardized conditions. The experiments were approved by the Ethics Committee of the Medical University Vienna and performed with the license of the Austrian Ministery for Science and Research.

\section{Induction of experimental autoimmune encephalomyelitis} (EAE) and tissue preparation

$\mathrm{T}$ cell lines against myelin basic protein (MBP, from guinea pig, Sigma), myelin oligodendrocyte glycoprotein (MOG, recombinant $\mathrm{N}$-terminal peptide $1-125$, rat, own production) and the astrocytic $\mathrm{Ca}^{2+}$ binding protein $\mathrm{S} 100 \beta$ (bovine, Sigma) were intraperitoneally injected to induce
Table 1 Characteristics of the NMO and MS tissues used

\begin{tabular}{|c|c|c|}
\hline $\begin{array}{c}\text { Disease } \\
\text { case-block }\end{array}$ & Activity & Region \\
\hline NMO 1-1 & LA & Cerebellum, medulla oblongata \\
\hline NMO 1-2 & EA & Spinal cord \\
\hline NMO 1-3 & LA & Spinal cord \\
\hline NMO 1-4 & EA & Spinal cord \\
\hline NMO 1-5 & LA & Mesencephalon \\
\hline NMO 1-6A & LA & Periventricular (4th ventricle) \\
\hline NMO 1-10A & LA & Temporal lobe \\
\hline NMO 2-49 & Acute, EA & Optic nerve \\
\hline NMO 3-2 & Chronic, IA & Spinal cord \\
\hline NMO 4-49 & Subacute/chronic LA-IA & Spinal cord \\
\hline NMO 5-2 & $E A, L A$ & Medulla oblongata \\
\hline NMO 6-2 & LA & Spinal cord \\
\hline NMO 6-4 & IA & Medulla oblongata \\
\hline AMS 1-1A & $E A, L A, I A$ & Spinal cord \\
\hline AMS 1-10D & $E A, L A, I A$ & Temporal lobe \\
\hline SPMS 1-12 & SEL, IA & Optic nerve \\
\hline SPMS 1-10 & SEL IA & Medulla \\
\hline SPMS 2-19 & SEL IA & Spinal cord \\
\hline SPMS 2-20 & SEL, IA & Spinal cord \\
\hline SPMS 2-21A & SEL, IA & Spinal cord \\
\hline AMS 6-15 & IA & Spinal cord \\
\hline SPMS 3-31 & SEL, IA & Medulla oblongata \\
\hline AMS 2-6 & EA & Brain \\
\hline RRMS 1 & $E A, L A$ & Brain \\
\hline AMS 3-5 & $E A, L A$ & Brain \\
\hline AMS 4-2 & $E A, L A$ & Brain \\
\hline AMS 5-97 & $E A, L A$ & Brain \\
\hline
\end{tabular}

Active lesions were identified and staged based on their dense infiltration by macrophages/activated microglia, and, in the case of NMO, by the presence of large numbers of neutrophilic granulocytes. Macrophages contained either early myelin degradation products (i.e. they were MOG+; early active lesions (EA)) or late myelin degradation products (i.e. they were PLP+; late active lesions (LA)). Inactive lesions (IA) had sharp lesion borders without macrophage infiltration of microglia activation, and slowly expanding lesions (SEL) were classified according to their inactive center, surrounded by a rim of activated microglia with some macrophages with myelin degradation products at the lesion margin.

EAE. These $\mathrm{T}$ cell lines have been established and expanded under conditions favoring TH1 cells, but not TH17 cells. Weight loss as earliest clinical sign of EAE started 4 days after the transfer. At this time point the animals received an intra-peritoneal injection with $1 \mathrm{ml}$ phosphate buffered saline (PBS) containing either $10 \mathrm{mg}$ NMO-IgG [5] or $10 \mathrm{mg}$ normal human IgG $\left(\right.$ Subcuvia $\left.^{\mathrm{R}}\right)$ [5]. 24 hours later, the animals were sacrificed with $\mathrm{CO}_{2}$ and perfused with $4 \%$ phosphate buffered paraformaldehyde (PFA). Brains and spinal cords were dissected, immersed for another 18 hours in PFA, and embedded in paraffin. 
The production of $\mathrm{T}$ cells transduced with green fluorescent protein (GFP), EAE induction with these cells, their re-isolation from CNS lesions and their characterization by flow cytometry followed established procedures [12,13].

\section{Determination of IFN- $\gamma$ production by polymerase chain reaction}

RNA was purified using the RNeasy kit with QIAshredders (Qiagen), converted to cDNA, and subjected to real-time polymerase chain reactions (RT-PCR) using the Power $\mathrm{SYBR}^{\circ}$ Green PCR Master Mix in a StepOnePlus RT-PCR system (both from Applied Biosystems) according to the manufacturers' instructions. A $25 \mu \mathrm{l}$ reaction mixture was used for each sample, containing cDNA and primer sets (200 nM) for IFN- $\gamma$ (forward: 5' - ATTCATGAGCATCG CCAAGTTC-3', reverse:

5'-TGACAGCTGGTGAATCACTCTGAT-3'; available at Real-Time Primer and Probe Database, RTPrimerDB ID:3773; https://medgen.ugent.be/rtprimerdb/; [14]) or beta actin (Actb; forward: 5' -AGGCCAACCGTGAAA AGATG-3'; reverse: 5'-ACCAGAGGCATACAGGGA CAA-3'; [15]). The initial 10-min denaturation step at $95^{\circ} \mathrm{C}$ was followed by 40 cycles of denaturation $\left(95^{\circ} \mathrm{C}\right.$, $15 \mathrm{~s})$ and annealing/extension $\left(60^{\circ} \mathrm{C}, 1 \mathrm{~min}\right)$. The absence of non-specific amplification was determined by melt curve analysis. All reactions were run in duplicates.

\section{Immunohistochemistry}

All tissue blocks were cut and stained as described [5], using the following antibodies: W3/13 (T-cells and granulocytes; mouse monoclonal; Serotec, UK); ED1 (macrophages, microglia; mouse monoclonal; Serotec, UK); commercial anti-AQP4 (rabbit polyclonal; Sigma, Germany); anti-GFAP (rabbit polyclonal; Dako, Denmark, or mouse monoclonal; Neomarkers, USA); anti-human immunoglobulin (biotinylated donkey; polyclonal; Amersham, UK) and anti-complement C9 (rabbit polyclonal [16]). Immunohistochemistry was completed by using appropriate biotinylated secondary antibodies (sheep anti-mouse, donkey anti-rabbit, donkey anti-sheep/goat; all from GE Healthcare/Amersham) and subsequent incubation of the sections with peroxidase-labelled avidin (Sigma, Germany).

For double immunostainings of OX40 and T cell markers, sections were incubated with anti-OX40 (1:30, Pharmingen) overnight at $4^{\circ} \mathrm{C}$, washed, further reacted with biotinylated donkey-anti-rabbit antibodies (1:500 Jackson ImmunoResearch, West Grove PA, USA) for 1 hour at RT, incubated with avidin-alkaline phosphatase (Sigma, Germany) and developed with Fast Blue (FB, Sigma, Germany) substrate. To retrieve CD4 or CD8 epitopes and inactivate the binding properties of the first round of antibodies (antiOx40 and biotinylated anti-rabbit), sections were steamed in a food steaming device in TRIS buffer containing EDTA (10 mM, pH = 9.0) for 90 minutes. Then, anti-CD4 (Ab8,
Neomarkers, 1:100 plus 1 F6, Acris, 1:500) or anti-CD8 antibodies (SP16, Neomarkers, 1:250) were applied overnight at $4^{\circ} \mathrm{C}$, followed by washing and incubation with biotinylated donkey-anti-rabbit antibodies and tyramide enhancement [17]. Finally, avidin-peroxidase (Sigma) was applied and sections were developed with aminoethyl carbazole (AEC, Sigma).

For double immunostainings of proliferating cell nuclear antigen (PCNA) and OX40, sections were steamed in citrate buffer for 30 minutes. Then, mouse anti-PCNA (clone PC10, Dako, 1:50000) was applied overnight at $4^{\circ} \mathrm{C}$. The sections were washed, incubated with biotinylated anti-mouse antibodies (Jackson ImmunoResearch, West Grove PA, USA, 1:500) for 1 hour, washed again, incubated with avidin-alkaline phosphatase (Sigma, Germany) and developed with Fast Blue (FB, Sigma, Germany) substrate. Afterwards, the sections were steamed for 45 minutes with TRIS buffer containing EDTA (10 mM, pH =9.0) to destroy free binding sites of the biotinylated anti-mouse antibodies used before, incubated with anti-OX40 (1:1000) overnight at $4{ }^{\circ} \mathrm{C}$, washed, further reacted with biotinylated anti-mouse antibodies (1:500) for 1 hour at RT and tyramide enhancement [17]. Finally, avidin-peroxidase (Sigma) was applied and sections were developed with aminoethyl carbazole (AEC, Sigma).

For fluorescent double labeling, Ox40 (mouse antibody) and anti-CD3 (rabbit antibody) were applied simultaneously at $4^{\circ} \mathrm{C}$ overnight. After washing with PBS, goatanti-rabbit Cy3 (Jackson ImmunoResearch, West Grove, Philadelphia; 1:200) and biotinylated anti-mouse (Amersham Pharmacia Biotech; 1:200) antibodies were applied simultaneously for 1 hour at RT. The staining was finished by application of streptavidin-Cy2 (Jackson ImmunoResearch; 1:75) for 1 hour at RT. Fluorescent preparations were examined using a confocal laser scan microscope (Leica SP5, Leica Mannheim, Germany). Scanning for Cy2 (488 nm) und Cy3 (543 nm) was performed sequentially to rule out fluorescence bleed-through.

\section{Microglia cultures and gene expression profiling upon IFN- $\boldsymbol{\gamma}$-treatment}

Microglia cultures were essentially produced as described [18] and had a purity of $\sim 99 \%$. They were treated with

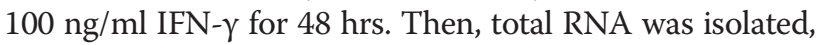
the mRNA transcribed to cDNA and sent to ImaGenes (Berlin, Germany) for gene expression studies, using $4 \times 44$ $\mathrm{K}$ Multiplex whole rat genome microarrays (Agilent G4131F). The raw data were subjected to quantile normalizations prior to comparison between groups and calculation of fold changes in expression.

\section{Statistical evaluation}

Statistical evaluations were performed using the PASW statistics 18 software system (SPSS Inc., Chicago, USA). 


\section{Results}

Active NMO lesions contain activated $\mathrm{CD}^{+}{ }^{+} \mathrm{T}$ cells $\mathrm{CD}^{+} \mathrm{T}$ cells expressing the activation markers OX40 and PCNA are found in NMO lesions, independent of their location within the neuraxis (Figures 1 and 2). The numbers of $\mathrm{Ox} 40^{+} \mathrm{T}$ cells were significantly higher in early active than in inactive NMO lesions (Figure 3), and in early active NMO compared to early active MS. Moreover, also the total numbers of $\mathrm{T}$ cells in early active $\mathrm{NMO}$ lesions were significantly higher than in inactive NMO lesions, while there was no statistically significant difference in $\mathrm{T}$ cell numbers between early active, inactive, or slowly expanding MS lesions (Figure 3). What is the relevance of this finding for the formation of astrocyte-destructive lesions in NMO?

T cells with different CNS antigen-specificities are differentially activated within the CNS

To address this question, we studied the extent of $\mathrm{T}$ cell activation in EAE provoked by $\mathrm{T}$ cells with different CNS antigen-specificities. T cells specific for MBP, S100 $\beta$, and MOG were labeled with GFP as described [12], and used for the induction of EAE. At the peak of EAE, we re-isolated these cells from the CNS, and studied their surface expression of the CD3-T cell receptor (TCR) complex, the IL-2R, and the Ox40 antigen as surrogate markers of $\mathrm{T}$ cell activation [12]. We found that MBPspecific $\mathrm{T}$ cells were strongly activated in the CNS, as indicated by a down-modulation of the TCR, and by increased levels of IL-2R and the Ox40 antigen. S100 $\beta$ specific $\mathrm{T}$ cells had an intermediate activation status, as revealed by unchanged expression levels of the TCR, by marginally increased levels of IL-2R and moderately increased levels of the Ox40 antigen. MOG-specific T cells, finally, did not show any increase in IL-2R and Ox40 antigen expression, and were hence not significantly activated within the CNS (Figure 4, Additional file 1: Figure S1). This is in marked contrast to the situation in vitro, where all these cells can be readily activated and expanded in response to their cognate antigen in the context of rat MHC class II products ([12], own observation).

T cells which are differently activated in the CNS differ in their ability to induce astrocyte-destructive lesions in NMO-IgG seropositive rats

We then used $\mathrm{T}$ cells which are differently activated in the CNS, i.e. $\mathrm{T}$ cells specific for MBP-, S100 $\beta$-, and MOG to induce EAE prior to the transfer of NMO-IgG. We found that all EAE models had similar NMO-IgG titres in the serum (between 1:320 and 1:1280; titres evenly distributed between the different groups),, that all $\mathrm{T}$ cells entered the CNS (Figure 5) and opened the blood-brain barrier for the entry of immunoglobulins (Figure 6). However, there were profound differences in the extent of astrocyte destruction. MBP-specific T cells induced the highest numbers of and the largest lesions

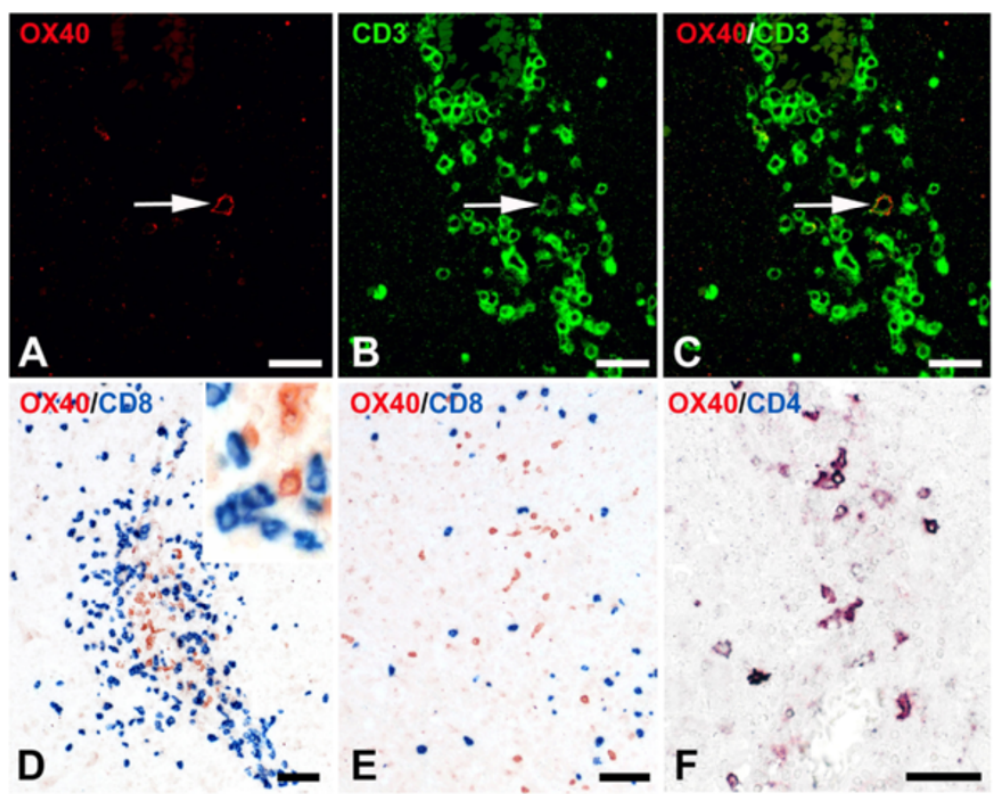

Figure 1 Ox40 expression by T cells in NMO lesions. (A-C) Confocal microscopy of a perivascular early active NMO lesion stained with antibodies against Ox40 (A, red) and CD3 (B, green) (overlay $\mathbf{C}$, yellow). The Ox40 antigen is expressed by a subset of T cells (white arrow). (D-F): Further stainings of NMO lesions with antibodies against Ox40 (brown) and CD8 (blue) (D-E) or CD4 (blue, F) reveals a complete absence of Ox40 expression on $\mathrm{CD}^{+} \mathrm{T}$ cells, both in perivascular lesions (D) and in the parenchyma at the edge of a demyelinating lesion (E). In contrast, Ox40 products are expressed by CD4 ${ }^{+} \mathrm{T}$ cells (purple), as seen in this perivascular lesion. Bars: $25 \mu \mathrm{m}$ (A-C) and $50 \mu \mathrm{m}$ (D-F). 


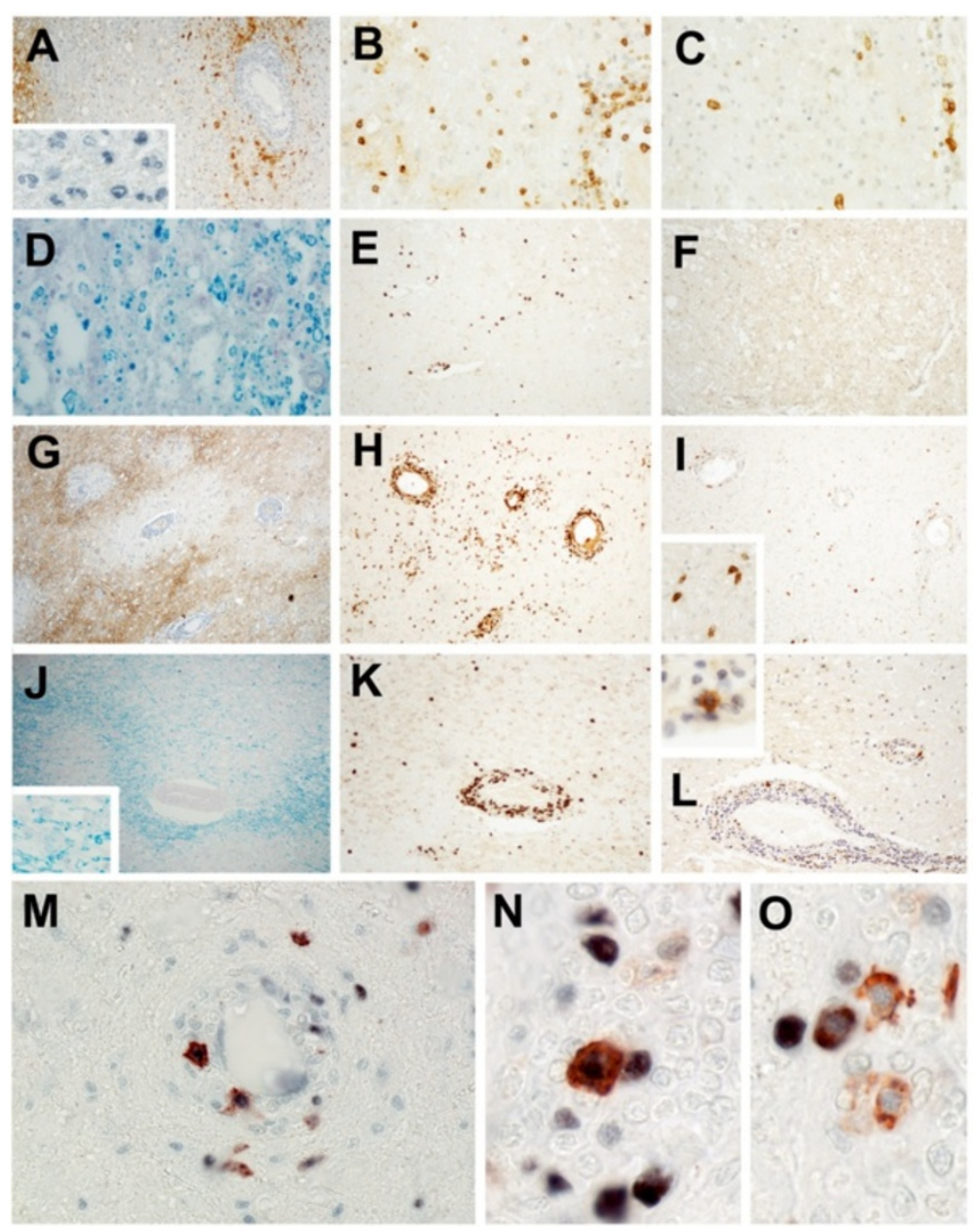

Figure 2 Differences in Ox40 expression by $\mathrm{CD}^{+} \mathrm{T}$ cells between NMO and MS lesions. Inflammatory lesions derived from spinal cords of NMO $(\mathbf{A}, \mathbf{B}, \mathbf{C})$ and $M S$ patients $(\mathbf{D}, \mathbf{E}, \mathbf{F})$, and from brains of NMO $(\mathbf{G}, \mathbf{H}, \mathbf{I})$ and $M S$ patients $(\mathbf{J}, \mathbf{K}, \mathbf{L})$ were reacted with antibodies specific for AQP4 (brown reaction product; counterstaining with hematoxylin reveals nuclei in blue; $\mathbf{A}, \mathbf{G}$ ), CD3 (brown reaction product; $\mathbf{B}, \mathbf{E}, \mathbf{H}, \mathbf{K}$ ), Ox40 (brown reaction product; $\mathbf{C}, \mathbf{F}, \mathbf{I}, \mathbf{L})$ or were stained with Kluever-Barrera to reveal myelin (blue; $\mathbf{D}, \mathbf{J})$. The spinal cord lesions shown are early active, as revealed by the large number of granulocytes in NMO (inlay in $\mathbf{A}$ ) and by the presence of myelin degradation products in macrophages in MS (D). The brain lesions shown are late active in $\mathrm{NMO}$, and early active, as revealed by macrophages containing myelin degradation products in MS (inlay in $\mathbf{J}$ ). The inlays in I and $\mathrm{L}$ show $\mathrm{Ox} 40^{+} \mathrm{T}$ cells. In early active NMO lesions, $18 \%$ of all perivascular and $24 \%$ of all parenchymal OX40 ${ }^{+}$cells also express the proliferating cell nuclear antigen PCNA (M-O; PCNA visualized by the dark blue, Ox 40 by the brown reaction product).

with AQP4 loss (Figure 7). Also S100 $\beta$-specific T cells facilitated the formation of astrocyte-destructive lesions, but the number of these lesions was significantly lower than the one found after transfer of MBP-specific T cells. In addition, there was a trend towards smaller lesions with AQP4-loss (Figure 7). Finally, MOG-specific T cells also infiltrated the CNS parenchyma, but did not induce astrocyte-destructive lesions (Figure 7). What are the differences between these T cells?

\section{Differences in T cell activation within the CNS translate into differences in the recruitment of macrophages/activated microglial cells}

Differences in $\mathrm{T}$ cell activation have consequences for the expression of chemokines responsible for the recruitment of macrophages/activated microglial cells [12]. We therefore studied the numbers of these cells in the inflamed spinal cords of NMO-IgG seropositive hosts. We found that MBP-specific $\mathrm{T}$ cells recruited the highest numbers of activated microglial cells/macrophages, S100 $\beta$-specific $\mathrm{T}$ cells intermediate numbers, and MOG-specific T cells the lowest numbers (medians 1464, 167, and $39 \mathrm{ED1}^{+}$cells $/ \mathrm{mm}^{2}$ of spinal cord cross sections, respectively; Figure 5).

Differences in T cell activation within the CNS translate into differences in IFN- $\gamma$ production, which affects the microglial expression of complement factors/inhibitors and of Fcgr3 Since EAE and NMO/EAE in Lewis rats are $\mathrm{T}_{\mathrm{H}} 1$-driven diseases with IFN- $\gamma$ as lead cytokine, we next determined the IFN- $\gamma$ expression of the re-isolated MBP-, S100ß-, 


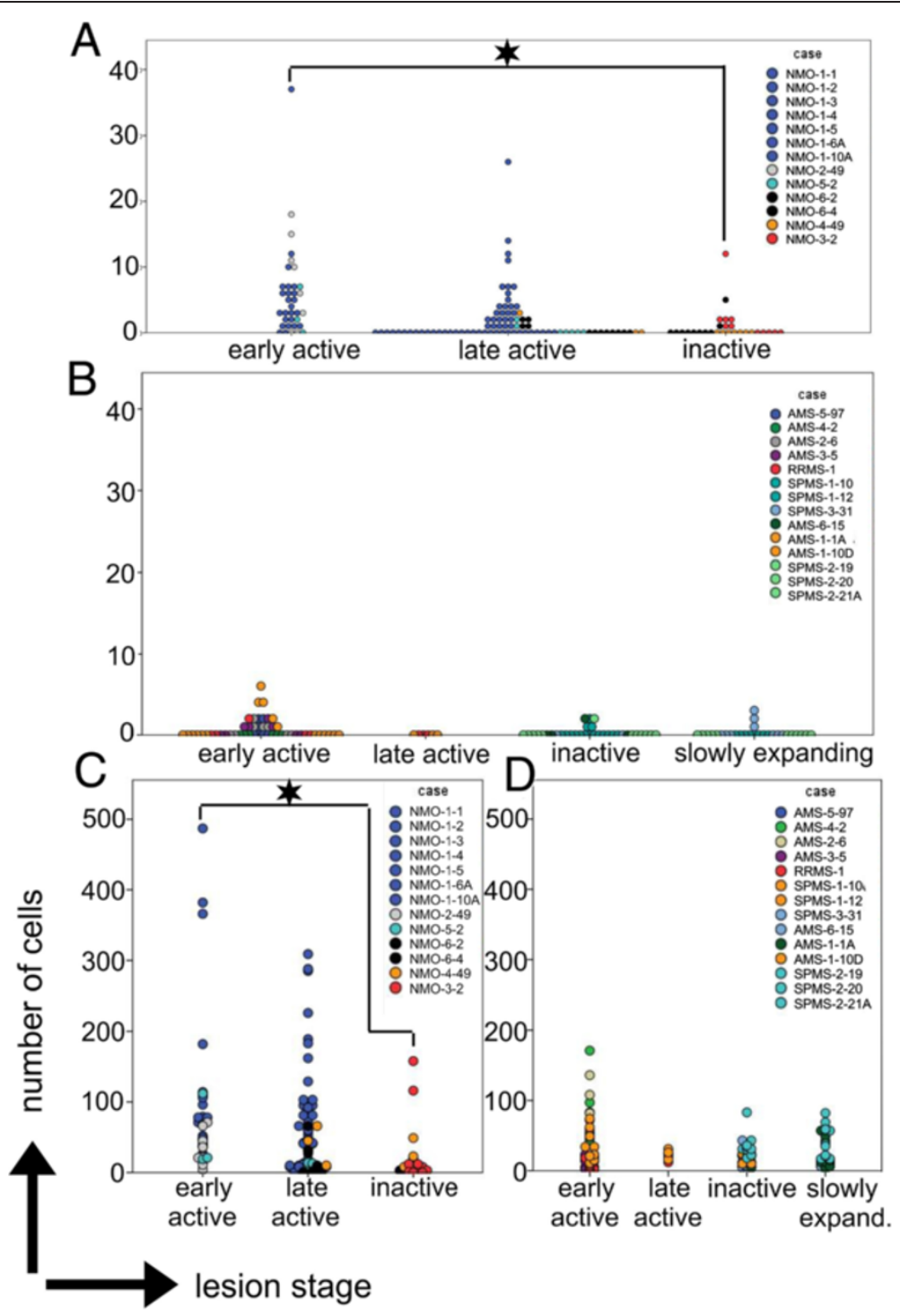

Figure 3 Numbers of $\mathrm{Ox} 40^{+}$and $\mathrm{CD}^{+} \mathrm{T}$ cells in $\mathrm{NMO}$ and MS lesions at different lesion stages. The number of $\mathrm{O} \times 40(\mathrm{~A}, \mathrm{~B})$ and $\mathrm{CD} 3$ $(\mathbf{C}, \mathbf{D})$ positive T cells of $7 \mathrm{NMO}(\mathbf{A}, \mathbf{C})$ and $9 \mathrm{MS}(\mathbf{B}, \mathbf{D})$ cases was determined by evaluating regions of interest in $\mathrm{NMO}(\mathrm{ROI} n=144)$ and $M S$ $(\mathrm{ROI} n=112)$ (each region $\left.=390.000 \mathrm{\mu m}^{2}\right)$ in spinal cord and brain lesions/inflamed parenchyma. Asterisks indicate statistically significant $(p<0,05$; Mann Whitney $U$ test with asymptotic significance (2 tailed)) differences between early active $(n=35)$ and inactive $(n=29)$ NMO lesions in the numbers of $O \times 40^{+} T$ cells $(\mathbf{A})$ and $\mathrm{CD}^{+} T$ cells $(\mathbf{C})$. The differences between early active $(n=51)$ and inactive $(n=34)$ MS lesions in the numbers of $\mathrm{Ox} 40^{+} T$ cells (B) and $\mathrm{CD}^{+} T$ cells (D) were not significant $(p=0,57$ and $p=0,107$, respectively). Please note that the differences in numbers of Ox40 $T$ cells and CD3 $3^{+}$cells between early active NMO $(n=35)$ and early active MS $(n=51)$ is also highly significant $(p<0,0001$ and $p=0,000318$, respectively).

and MOG-specific T cells by RT-PCR, and observed that MBP-specific T cells had significantly higher relative IFN- $\gamma$ mRNA expression levels than their S100ß- or MOGspecific counterparts (Figure 8). We next stimulated microglia with IFN- $\gamma$ or vehicle (control) in vitro, and studied the IFN- $\gamma$ induced changes in the expression of transcripts encoding complement proteins and Fcgr3. We observed a profound upregulation of complement component $1, \mathrm{r}$ subcomponent $(\mathrm{C} 1 \mathrm{r})$, complement component 1 , q subcomponent, $\mathrm{C}$ chain $(\mathrm{C} 1 \mathrm{qc})$, complement component
2 (C2), complement component $3(\mathrm{C} 3)$, complement component $6(\mathrm{C} 6)$, and complement factor $\mathrm{B}(\mathrm{Cfb})$, coinciding with the downregulation of the inhibitory complement factor $\mathrm{H}(\mathrm{CfH})$ and of "similar to complement factor $\mathrm{H}$-related protein" (sim. to $\mathrm{CfH}$ ). We also found a profound upregulation of Fcgr3 (Figure 8).

\section{Discussion}

Although $\mathrm{T}$ cells are regularly found in NMO lesions, our knowledge about these cells is rather limited. For 


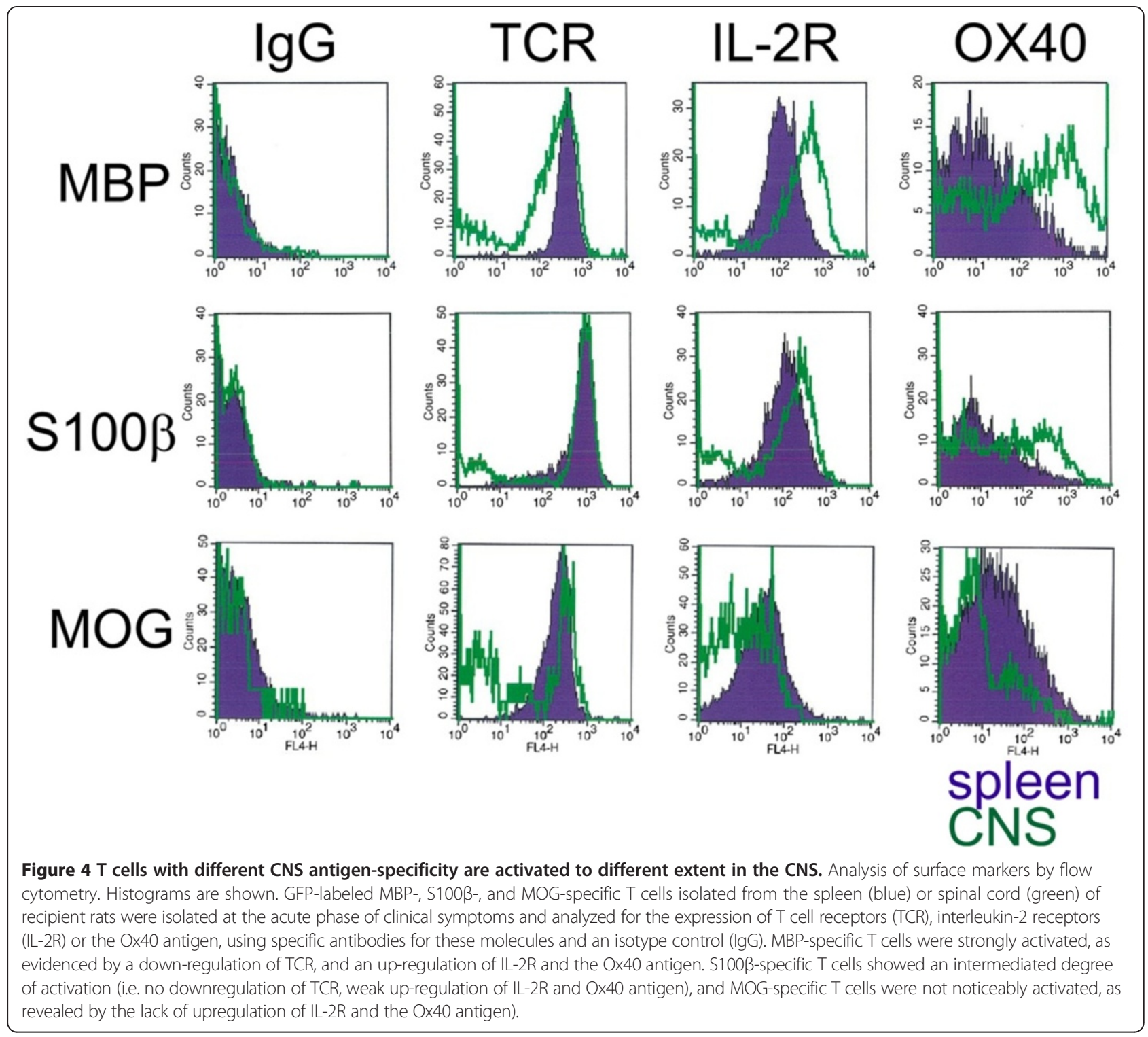

example, we do not know yet whether these cells recognize AQP4 [21] or other CNS antigens, and whether these cells initiated the lesions or were non-specifically recruited to these sites. However, we learned from experimental models of MS or NMO that only migration-competent CNS antigen-specific T cells can cross through the brain capillary endothelium, open the blood-brain barrier for the subsequent entry of additional inflammatory mediators, and initiate inflammatory CNS lesions. This immigration phase is similar in all CNS antigen-specific $\mathrm{T}$ cells, irrespective of their pathogenicity [12]. However, only highly pathogenic T cells are subsequently activated within the CNS [12]. We show here that the extent of $\mathrm{T}$ cell activation within the tissue is a critical parameter for the formation of astrocyte-destructive lesions in NMO-IgG seropositive rats, and we provide evidence for the presence of activated $\mathrm{CD} 4^{+} \mathrm{T}$ cells in CNS lesions of NMO patients, suggesting that these lesions have been initiated by pathogenic $\mathrm{T}$ cells recognizing antigens in the CNS.

Full activation of T cells in the CNS - as exemplified in our study by MBP-specific T cells - translates into the profound up-regulation of Ox40, increased levels of IL-2R, down-modulation of TCR, and robust production of IFN- $\gamma[12,22,23]$. Engagement of Ox40 with its ligand supports the survival of $\mathrm{T}$ cells and augments antigendriven TCR-signaling in these cells [24]. Interactions of Ox40 with Ox40L on the surface of antigen presenting cells enhances their production of pro-inflammatory cytokines like IL-1 beta or IL- 6 and their surface expression of co-stimulatory molecules $[25,26]$. Interestingly, IFN- $\gamma$ triggers the expression of Ox40L in microglia [27] and 


\section{A}
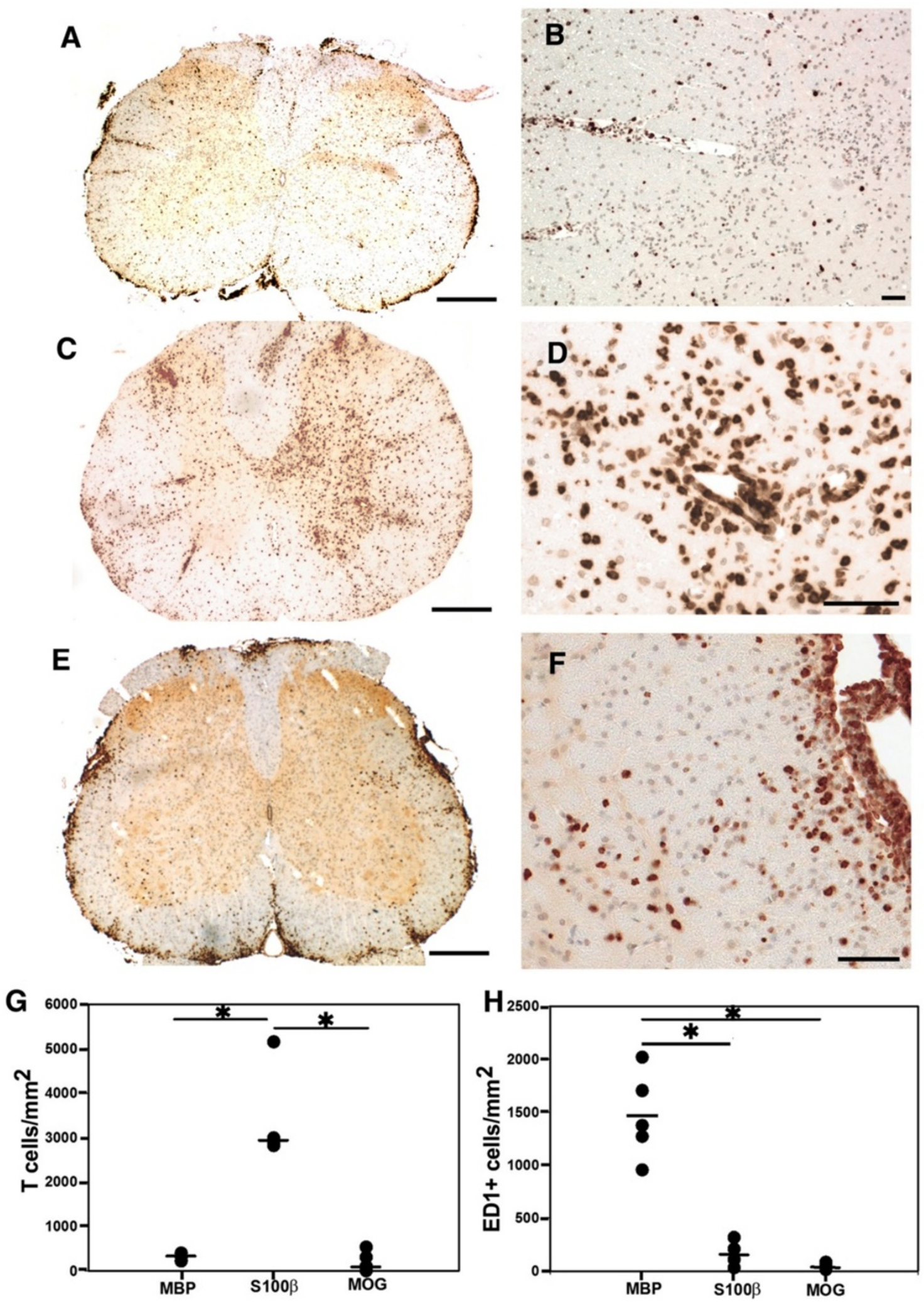

Figure $\mathbf{5}$ (See legend on next page.) 
(See figure on previous page.)

Figure $5 \mathrm{~T}$ cells infiltration of the spinal cord following the initiation of NMO-like lesions in NMO-IgG seropositive animals by $\mathrm{T}$ cells with different CNS antigen-specificities. (A-F) T cells specific for MBP (A,B), S100ß $(\mathbf{C}, \mathbf{D})$ and MOG $(\mathbf{E}, \mathbf{F})$ were used to induce CNS

inflammation, followed by transfer of NMO-IgG 4 days later. The animals were sacrificed 5 days after $\mathrm{T}$ cell transfer. For histological evaluation, their spinal cords were reacted with anti-CD3 antibodies (brown reaction product) and counterstained with hematoxylin to reveal nuclei (blue). bars $=500 \mu \mathrm{m}(\mathbf{A}, \mathbf{C}, \mathbf{E})$ and $100 \mu \mathrm{m}(\mathbf{B}, \mathbf{D}, \mathbf{F})$. (G) The average number of T cells per $\mathrm{mm}^{2}$ of lesions was determined by evaluating 5 representative spinal cord cross sections (1 cervical, 2 thoracal, 2 lumbar cross sections) per animal, using 5 animals (MBP- and MOG-specific T cells) or 4 animals ( $\$ 100 \beta$-specific T cells) per group. Asterisks indicate statistically significant $(p<0,05)$ differences between individual CNS antigen specificities of the T cells used to induce CNS inflammation (Kruskal-Wallis followed by Mann-Whitney U test and Bonferroni-Holm correction; $p=0,0476$ for MBP/ S100ß and S100B/MOG, $p=0.858$ for MBP/MOG). (H) Numbers of ED $1^{+}$cells (activated microglia/macrophages) in spinal cord cross sections. The cell numbers were determined by evaluating one complete spinal cord cross section per animal, using 5 animals (MBP- and MOG-specific T cells) or 4 animals (S100ß-specific T cells) per group. Asterisks indicate statistically significant $(p<0,05)$ differences between individual CNS antigen specificities of the T cells used to induce CNS inflammation (Kruskal-Wallis followed by Mann-Whitney $U$ test and Bonferroni-Holm correction; $p=0,048$ for MBP/ S100 $\beta, p=0,024$ for MBP/MOG, and $p=0,189$ for S100ß/MOG).

induces a microglial phenotype characterized by the up-regulation of complement factors and the downregulation of complement inhibitors which could favor the antibody and complement mediated destruction of astrocytes in experimental NMO lesions. IFN- $\gamma$ also induces the upregulation of Fcgr3, which plays an important role in antibody-dependent cell-mediated cytotoxicity (ADCC). The binding of the antibody Fc region to Fc gamma receptors on activated microglia/macrophages

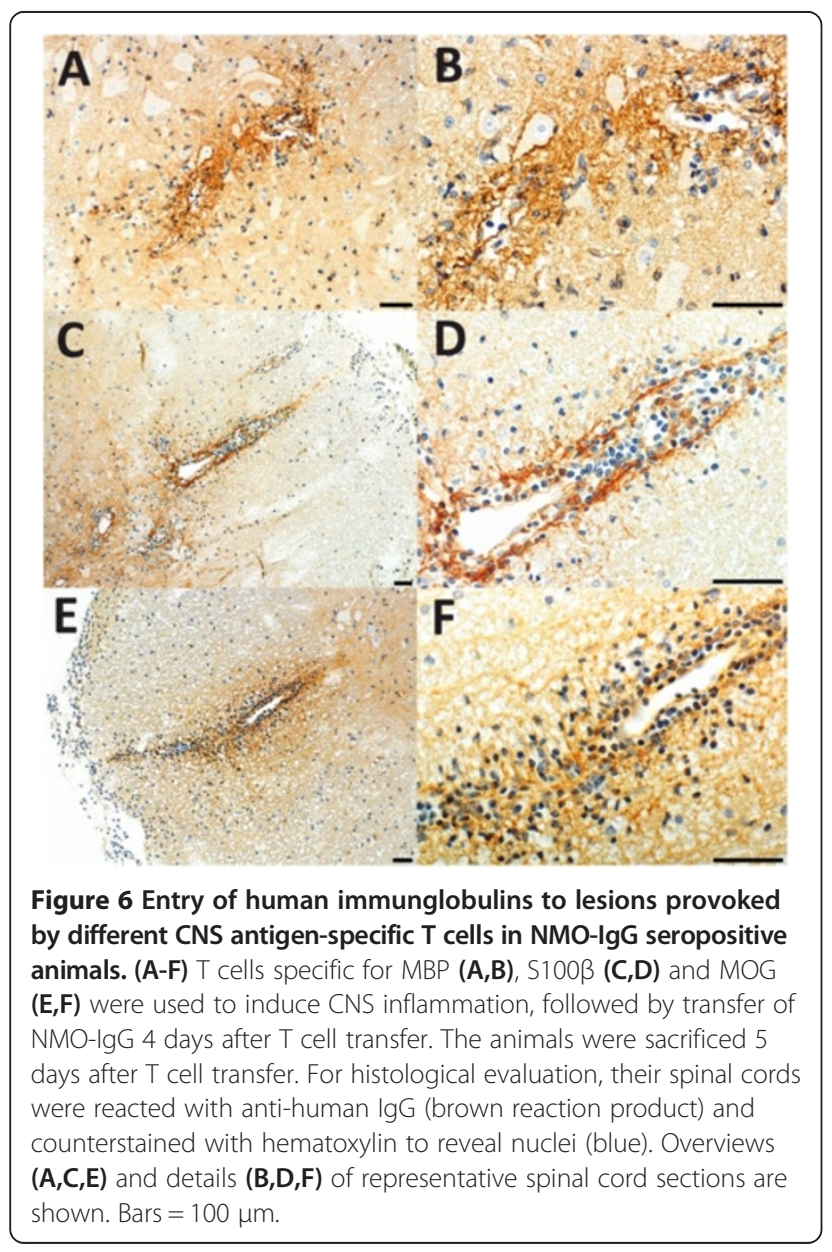

promotes their accumulation and plays an important role in NMO pathology, as evidenced by a reduction of tissue damage in NMO models involving mice lacking Fcgr3, mice treated with Fcgr blockers, or mice containing mutated NMO-IgG without ADCC function [28].

Fully activated T cells also produce CCL2 and CCL3 transcripts [12] which may further attract large numbers of macrophages/activated microglia to the lesions.

The intermediate activation of T cells - as exemplified by $\mathrm{S} 100 \beta$-specific $\mathrm{T}$ cells - recruits much less macrophages/activated microglial cells, which is in line with lower expression levels of CCL2 and CCL3 in these cells than in their MBP-specific counterparts [12]. This activation stage may still provide enough surface expression of Ox40 to augment antigen-driven TCR-signaling, thus enabling low doses of antigen to promote responses normally only induced by higher doses [29]. Intermediately activated S100 $\beta$-specific T cells only produce low amounts of IFN $-\gamma$, but may compensate for this by being present in the CNS parenchyma in high cell numbers. This could explain why S100ß-specific T cells are still able to facilitate the formation of astrocyte-destructive lesions in NMO-IgG seropositive animals.

However, if $\mathrm{T}$ cells were not activated at all in the CNS - as exemplified by MOG-specific T cells -, recruited macrophages/activated microglial cells in extremely low numbers, and did not compensate for low IFN- $\gamma$ production levels by high parenchymal cell numbers, astrocytedestructive lesions in the presence of NMO-IgG and complement were not formed despite inflammationinduced blood-brain barrier leakage.

Is there any evidence for the activation of AQP4specific $\mathrm{T}$ cells in NMO/EAE? This question could not yet be experimentally addressed, since all AQP4-specific rat $\mathrm{T}$ cells tested so far were only weakly encephalitogenic [22] and could not be re-isolated in sufficiently high numbers to test their surface expression of activation markers by FACS analysis, and since commercially available antibodies against the rat Ox40 antigen did not work in paraffin-embedded or frozen tissue. However, AQP4- 


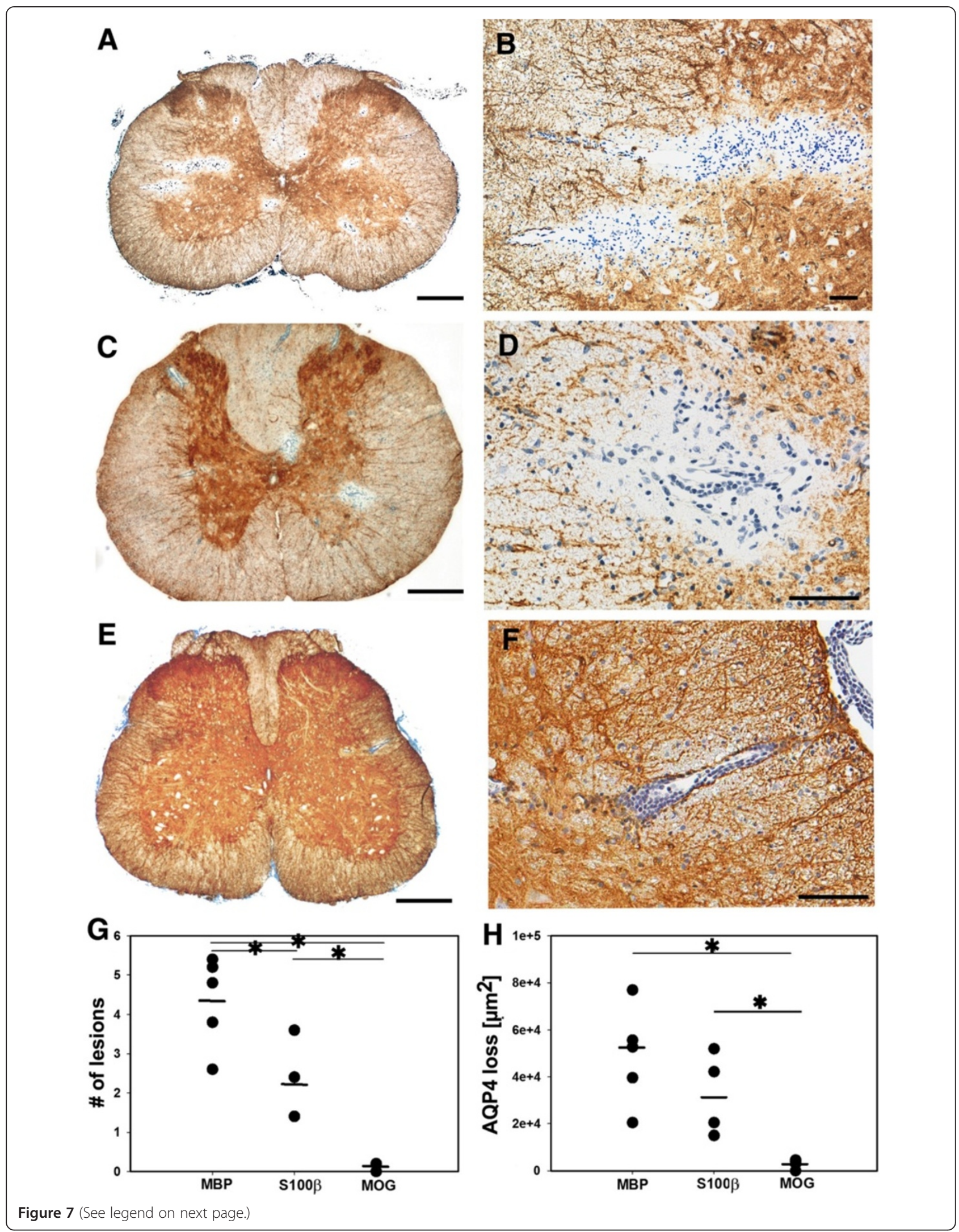


(See figure on previous page.)

Figure 7 Loss of AQP4 reactivity in NMO-like lesions initiated by T cells with different CNS antigen-specificities. (A-F) T cells specific for $\operatorname{MBP}(\mathbf{A}, \mathbf{B})$, S100ß $(\mathbf{C}, \mathbf{D})$ and MOG $(\mathbf{E}, \mathbf{F})$ were used to induce CNS inflammation, followed by transfer of NMO-lgG 4 days later. The animals were sacrificed 5 days after T cell transfer. For histological evaluation, their spinal cords were reacted with anti-AQP4 antibodies (brown reaction product) and counterstained with hematoxylin to reveal nuclei (blue). bars $=500 \mu \mathrm{m}(\mathbf{A}, \mathbf{C}, \mathbf{E})$ and $100 \mu \mathrm{m}(\mathbf{B}, \mathbf{D}, \mathbf{F})$. (G) The average number of lesions with AQP4 loss per spinal cord cross section, as determined by evaluating 5 representative spinal cord cross sections (1 cervical, 2 thoracal, and 2 lumbar cross sections) per animal, using 5 animals (MBP, MOG) and 4 animals (S100/) per group. Asterisks indicate statistically significant differences between individual CNS antigen specificities of the T cells used to induce CNS inflammation (ANOVA-Holm Sidak; $p<0,001$ for MBP-specific T cells compared to MOG-specific T cells; $p=0,008$ for MBP-specific T cells compared to S100ß-specific T cells; and $p=0,005$ for S100ß-specific T cells compared to MOG-specific T cells). (H) The largest lesion with AQP4 loss per animal, using 5 animals (MBP, MOG) and 4 animals (S100ß) per group. Asterisks indicate statistically significant differences between individual CNS antigen specificities of the T cells used to induce CNS inflammation (Mann-Whitney $U$ test with Bonferroni-Holm correction; $p=0,732$ for MBP/S100 $\beta, p=0,024$ for MBP/MOG, $p=0,048$ for S100 $/ M O G$ ).

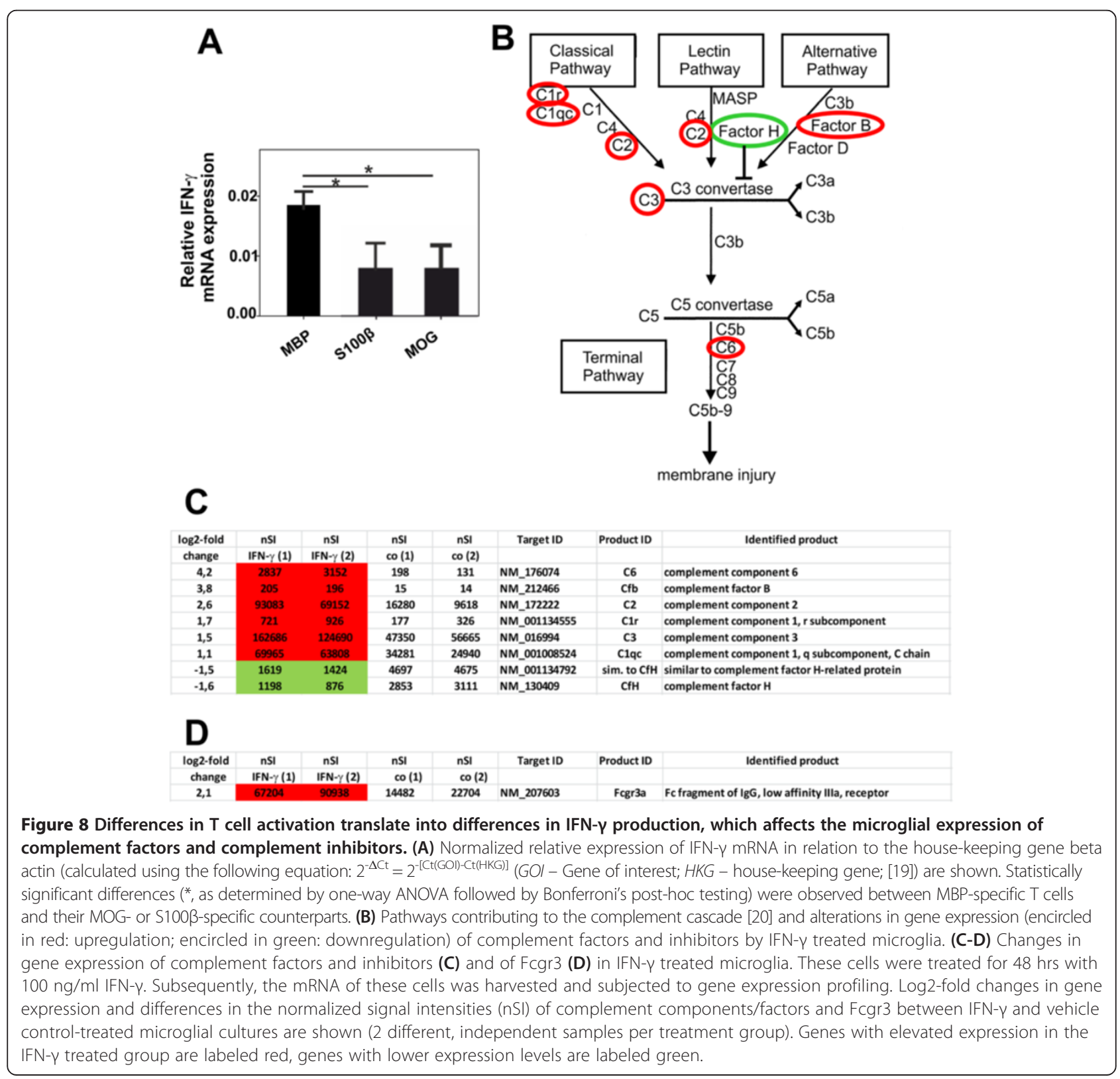


specific T cells cause lesions with AQP4 loss in NMO-IgG seropositive hosts [22], which suggests an activation of these cells within the CNS.

It might be argued that $\mathrm{NMO}$ is a $\mathrm{T}_{\mathrm{H}} 17$-driven disease [30,31], while $\mathrm{NMO} / \mathrm{EAE}$ is driven by $\mathrm{T}_{\mathrm{H}} 1 \mathrm{~T}$ cells [5,21], and that our findings about the consequences of activated, IFN- $\gamma$ producing cells might be irrelevant for NMO. However, human $\mathrm{T}_{\mathrm{H}} 17$ cells comprise a heterogeneous subset of cells including IFN- $\gamma$-producing cells [32-34] and have the ability to shift to $\mathrm{T}_{\mathrm{H}} 1$ cells [35].

It was unexpected to see significantly higher numbers of T-cells expressing the activation marker Ox40 in active NMO compared to active MS lesions. Since this was the case in early as well as late active lesions, and in brain as well as spinal cord lesions, this observation cannot simply be explained by stage or location dependent differences in the lesions. In NMO lesions, Ox40 expression is seen in $\mathrm{CD} 4^{+} \mathrm{T}$ cells, and it has been described that activated $\mathrm{CD} 4^{+} \mathrm{T}$ cells have prolonged Ox40 expression [24]. In MS lesions, however, the activated $\mathrm{T}$ cells might belong to the clonally expanded $\mathrm{CD} 8^{+} \mathrm{T}$ cell pool [36], and Ox40 expression in activated $\mathrm{CD} 8^{+} \mathrm{T}$ cells appears to be more transient compared to that in activated $\mathrm{CD} 4^{+}$ T cells [24]. Hence, Ox40 expression in MS lesions may escape detection.

\section{Conclusion}

In summary our data indicate that even in conditions of inflammation and blood-brain barrier damage alone, entry of AQP4 specific autoantibodies into the CNS is insufficient to trigger astrocyte destructive NMO lesions and that the additional activation of effector mechanisms in the lesions is necessary to facilitate complement-mediated and antibody-dependent cellular cytotoxicity. Such an environment is provided in $\mathrm{T}$ cell-mediated inflammation when invading $\mathrm{T}$ cells are antigen-specifically activated within the CNS. Our data further suggest that a similar mechanism may operate in human NMO lesions, since the infiltrating T-cells show the respective signs of activation. Whether these cells recognize AQP4 or other CNS antigens is not resolved yet, since $\mathrm{CD} 4^{+} \mathrm{T}$ cells specific for AQP4 [37,38] or PLP [37] have been observed in the peripheral blood of NMO patients.

\section{Additional file}

Additional file 1: Figure S1. T cells with different CNS antigen-specificity are activated to different extent in the CNS. Shown here are dotblots of an analysis of surface markers by flow cytometry. GFP-labeled MBP-, S100ß-, and MOG-specific T cells isolated from the spleen or spinal cord of recipient rats were isolated at the acute phase of clinical symptoms and analyzed for the expression of $\mathrm{T}$ cell receptors (TCR), interleukin-2 receptors (IL-2R) or the Ox40 antigen, using specific antibodies for these molecules and an isotype control (lgG). MBP-specific T cells were strongly activated, as evidenced by a down-regulation of TCR, and an up-regulation of IL-2R and the Ox40 antigen.
S100ß-specific T cells showed an intermediated degree of activation (i.e. no downregulation of TCR, weak up-regulation of IL-2R and Ox40 antigen), and MOG-specific $T$ cells were not noticeably activated, as revealed by the lack of upregulation of IL-2R and the Ox40 antigen).

\section{Competing interests}

The authors declare that they have no competing interests.

\section{Authors' contributions}

MP established T cell lines, performed the animal experiments and histologically analyzed all rat and human CNS tissues; NK established GFP-expressing T cell lines and characterized the re-isolated T cells by flow cytometry; JWE sorted the GFP-expressing T cells, JB established the OX40 stainings and made confocal microscopy, JM-S performed the PCNA studies, MK, RM and M-TF established microglia cultures, performed the treatments with IFN- $\gamma$ and analyzed microglia by real-time PCR (MK) and with microarrays (RM, M-TF). SM determined the anti-AQP4 antibody titres of the experimental animals. TM and KF provided the NMO-IgG preparation J0. HW and MR provided intellectual input. MB and HL designed the study and planned all experiments. All authors read and approved the final manuscript.

\section{Acknowledgements}

We thank Marianne Leisser and Angela Kury for excellent technical assistance.

\section{Funding}

This work was supported by the European Union (grant numbers LSHM-CT2005-018637 and PITN-GA-2012-316722), the Austrian Science Fund (FWF grant number P25240-B24 to MB and 1916-B13 (International Programme, Eugène Devic European Network) to $\mathrm{HL}$ and MR), the PhD programme Cell Communication in Health and Disease (CCHD, cofunded by the Austrian Science Fund and the Medical University Vienna), and a research grant (number 2007104) of the Interdisciplinary Center for Research and Treatment (IFTZ) of Innsbruck Medical University. It was supported in parts by grants-in-aid for Scientific Research of the Ministry of Education, Culture, Sports, Science and Technology (19209032, 20390241, 22229008), and the Health and Labour Sciences Research Grant for Neuroimmunological Diseases of the Ministry of Health, Labor and Welfare of Japan.

\section{Author details}

${ }^{1}$ Department of Neuroimmunology, Center for Brain Research, Medical University Vienna, Spitalgasse 4, Vienna A-1090, Austria. ${ }^{2}$ Max-Planck-Insitute for Neurobiology, Max-Lebsche-Platz 31, Munich 81377, Germany. ${ }^{3}$ Institute of Clinical Neuroimmunology, LMU Munich, Max-Lebsche Platz 31, Munich 81377, Germany. ${ }^{4}$ Clinical Department of Neurology, Innsbruck Medical University, Innrain 66/2, Innsbruck A-6020, Austria. ${ }^{5}$ Helmholtz Center Munich, Institute of Molecular Immunology, Marchioninistr. 25, Munich 81377, Germany. ${ }^{6}$ Departments of Multiple Sclerosis Therapeutics and Neurology, Tohoku University Graduate School of Medicine, 1-1 Seiryomachi, Aobaku, Sendai 980-8574, Japan.

Received: 20 December 2013 Accepted: 21 December 2013 Published: 24 December 2013

\section{References}

1. Fujihara K, Misu T, Nakashima I, et al: Neuromyelitis optica should be classified as an astrocytopathic disease rather than a demyelinating disease. Clin Exp Neuroimmunol 2012, 3:58-73.

2. Lennon VA, Kryzer TJ, Pittock SJ, Verkman AS, Hinson SR: IgG marker of optic-spinal multiple sclerosis binds to the aquaporin-4 water channel. J Exp Med 2005, 202:473-477.

3. Lennon VA, Wingerchuk DM, Kryzer TJ, et al: A serum autoantibody marker of neuromyelitis optica: distinction from multiple sclerosis. Lancet 2004, 364:2106-2112.

4. Bennett JL, Lam C, Kalluri SR, et al: Intrathecal pathogenic anti-aquaporin-4 antibodies in early neuromyelitis optica. Ann Neurol 2009, 66:617-629.

5. Bradl M, Misu T, Takahashi T, et al: Neuromyelitis optica: pathogenicity of patient immunoglobulin in vivo. Ann Neurol 2009, 66:630-643.

6. Nishiyama S, Ito T, Misu T, et al: A case of NMO seropositive for aquaporin-4 antibody more than 10 years before onset. Neurology 2009, 72:1960-1961. 
7. Saadoun S, Waters $P$, MacDonald C, et al: Neutrophil protease inhibition reduces neuromyelitis optica-immunoglobulin $\mathrm{G}$-induced damage in mouse brain. Ann Neurol 2012, 71:323-333.

8. Ratelade J, Bennett JL, Verkman AS: Intravenous neuromyelitis optica autoantibody in mice targets aquaporin-4 in peripheral organs and area postrema. PLoS One 2011, 6(11):e27412

9. Kitic M, Hochmeister S, Wimmer I, et al: Intrastriatal injection of interleukin 1 beta triggers the formation of neuromyelitis optica-like lesions in NMO-lgG seropositive rats. Acta Neuropathol Comm 2013, 1:5.

10. Lucchinetti CF, Mandler RN, McGavern D, et al: A role for humoral mechanisms in the pathogenesis of Devic's neuromyelitis optica. Brain 2002, 125:1450-1461.

11. Misu T, Höftberger R, Fujihara K, et al: Presence of six different lesion types suggests diverse mechanisms of tissue injury in neuromyelitis optica. Acta Neuropathol 2013, 125:815-827.

12. Kawakami N, Lassmann S, Li Z, et al: The activation status of neuroantigenspecific T cells in the target organ determines the clinical outcome of autoimmune encephalomyelitis. J Exp Med 2004, 199:185-197.

13. Kawakami N, Odoardi F, Ziemssen T, et al: Autoimmune CD4+ T cell memory: lifelong persistence of encephalitogenic $T$ cell clones in healthy immune repertoires. J Immunol 2005, 175:69-81.

14. Pattyn F: RTPrimerDB: the Real-Time PCR primer and probe database. Nucleic Acid Res 2003, 31:122-123.

15. Gayle DA: Maternal LPS induces cytokines in the amniotic fluid and corticotropin releasing hormone in the fetal rat brain. Am J Physiol Regul Integr Comp Physiol 2004, 286:R1024-R1029.

16. Piddlesden SJ, Lassmann $H$, Zimprich F, Morgan BP, Linington C: The demyelinating potential of antibodies to myelin oligodendrocyte glycoprotein is related to their ability to fix complement. Am J Path 1993, 143:555-564.

17. King G, Payne S, Walker F, Murray Gl: A highly sensitive detection method for immunohistochemistry using biotinylated tyramine. J Path 1997, 183:237-241.

18. Hochmeister S, Zeitelhofer M, Bauer J, et al: After injection into the striatum, in vitro-differentiated microglia- and bone marrow-derived dendritic cells can leave the central nervous system via the blood stream. Am J Path 2008, 173:1669-1681.

19. Livak KJ, Schmittgen TD: Analysis of relative gene expression data using real-time quantitative $P C R$ and the $2-\Delta \Delta C T$ method. Methods 2001 25:402-408

20. Zhou W, Marsh JE, Sacks SH: Intrarenal synthesis of complement. Kidney Intern 2001, 59:1227-1235.

21. Pohl M, Fischer MT, Mader $\mathrm{S}$, et al: Pathogenic T cell responses against aquaporin 4. Acta Neuropath 2011, 122:21-34.

22. Lathrop SK, Huddleston CA, Dullforce PA, Montfort MJ, Weinberg AD, Parker DC: A signal through OX40 (CD134) allows anergic, autoreactive T cells to acquire effector cell functions. J Immunol 2004, 172:6735-6743.

23. Williams CA, Murray SE, Weinberg AD, Parker DC: OX40-mediated differentiation to effector function requires IL-2 receptor signaling but not CD28, CD40, IL-12Rbeta2, or T-bet. J Immunol 2007, 178:7694-7702.

24. Croft M: Control of immunity by the TNFR-related molecule OX40 (CD134). Ann Rev Immunol 2010, 28:57-78.

25. Baumann R, Yousefi S, Simon D, Russmann S, Mueller C, Simon HU: Functional expression of CD134 by neutrophils. Eur J Immunol 2004, 34:2268-2275.

26. Ohshima Y, Tanaka Y, Tozawa H, Takahashi Y, Maliszewski C, Delespesse G: Expression and function of OX40 ligand on human dendritic cells. J Immunol 1997, 159:3838-3848.

27. Wang $Y$, Li M, Song M, et al: Expression of OX40 ligand in microglia activated by IFN-gamma sustains a protective CD4+ T-cell response in vitro. Cell Immunol 2008, 251:86-92.

28. Ratelade J, Asavapanumas N, Ritchie AM, Wemlinger S, Bennett JL, Verkman AS: Involvement of antibody-dependent cell-mediated cytotoxicity in inflammatory demyelination in a mouse model of neuromyelitis optica. Acta Neuropathol 2013. DOI: 10.1007/s00401-013-1172-z.

29. Rogers PR, Croft M: CD28, Ox40, LFA-1, and CD4 modulation of Th1/Th2 differentiation is directly dependent on the dose of antigen. $J$ Immunol 2000, 164:2955-2963.

30. Ishizu T, Osoegawa M, Mei FJ, et al: Intrathecal activation of the IL-17/IL-8 axis in opticospinal multiple sclerosis. Brain 2005, 128:988-1002.

31. Uzawa A, Mori M, Arai $K$, et al: Cytokine and chemokine profiles in neuromyelitis optica: significance of interleukin-6. Mult Scler 2010, 16:1443-1452
32. Annunziato F, Cosmi L, Santarlasci V, et al: Phenotypic and functional features of human Th17 cells. J Exp Med 2007, 204:1849-1861.

33. Boniface K, Blumenschein WM, Brovont-Porth K, et al: Human Th17 cells comprise heterogeneous subsets including IFN-gamma-producing cells with distinct properties from the Th1 lineage. J Immunol 2010, 185:679-687.

34. Zielinski CE, Mele F, Aschenbrenner D, et al: Pathogen-induced human $\mathrm{TH} 17$ cells produce IFN-gamma or IL-10 and are regulated by IL-1beta. Nature 2012, 484:514-518.

35. Annunziato F, Romagnani S: Do studies in humans better depict Th17 cells? Blood 2009, 114:2213-2219.

36. Babbe H, Roers A, Waisman A, et al: Clonal expansions of CD8(+) T cells dominate the $T$ cell infiltrate in active multiple sclerosis lesions as shown by micromanipulation and single cell polymerase chain reaction. $J$ Exp Med 2000, 192:393-404.

37. Matsuya N, Komori M, Nomura K, et al: Increased T-cell immunity against aquaporin-4 and proteolipid protein in neuromyelitis optica. Int Immunol 2011, 23:565-573.

38. Varrin-Doyer M, Spencer CM, Schulze-Topphoff U, et al: Aquaporin 4-specific $T$ cells in neuromyelitis optica exhibit a Th17 bias and recognize Clostridium ABC transporter. Ann Neurol 2012, 72:53-64.

doi:10.1186/2051-5960-1-85

Cite this article as: Pohl et al.: T cell-activation in neuromyelitis optica lesions plays a role in their formation. Acta Neuropathologica Communications 2013 1:85

\section{Submit your next manuscript to BioMed Central and take full advantage of:}

- Convenient online submission

- Thorough peer review

- No space constraints or color figure charges

- Immediate publication on acceptance

- Inclusion in PubMed, CAS, Scopus and Google Scholar

- Research which is freely available for redistribution 